\title{
The Effect of Tofogliflozin Treatment on Postprandial Glucose and Lipid Metabolism in Japanese Men With Type 2 Diabetes: A Pilot Study
}

\author{
Hirokazu Kakuda ${ }^{\text {a }}$, Junji Kobayashib, e, Masaru Sakuraic ${ }^{c}$, Masahiro Kakuda ${ }^{a}$, \\ Noboru Takekoshi ${ }^{\mathrm{d}}$
}

\begin{abstract}
Background: Postprandial hyperglycemia and hyperlipidemia are highly related to the development of atherosclerosis. Sodium/glucose cotransporter-2 (SGLT2) inhibitors have attracted attention as a new class of anti-diabetic agents for the treatment of type 2 diabetes. We investigated the effect of tofogliflozin on postprandial glucose and lipid metabolism in Japanese male patients with type 2 diabetes.
\end{abstract}

Methods: Ten Japanese men with type 2 diabetes (average age 66.3 years) were orally administered tofogliflozin (20 mg per day) for 8 weeks followed by a subsequent 8 weeks of washout of the agent. At 0,8 and 16 weeks, postprandial metabolic parameters were measured at 0,60 and $120 \mathrm{~min}$ after cookie ingestion.

Results: There were significant reductions in body weight and body mass index at 8 weeks. There was a reduction in HbA1c at 8 weeks, which returned to pretreatment levels at 16 weeks. Serum insulin levels did not change during the entire study period under either fasting or postprandial state. The area under the curve of plasma glucagon significantly increased at 8 weeks. There were no changes in lipid and lipoprotein levels either in fasting or postprandial state except for tendency toward reduction in postprandial triglycerides at 8 weeks and increase in HDL-C at 16 weeks.

Conclusions: Tofogliflozin treatment causes an improvement of postprandial glucose metabolism but not considerable postprandial lipid metabolism.

Keywords: Tofogliflozin; Sodium glucose cotransporter; Postprandial metabolism

Manuscript accepted for publication December 01, 2016

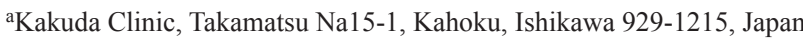
bDepartment of General Medicine, Kanazawa Medical University, Ishikawa, Japan

'Department of Hygiene, Kanazawa Medical University, Ishikawa, Japan

${ }^{\mathrm{d}}$ Kanazawa Medical University, Ishikawa, Japan

${ }^{e}$ Corresponding Author: Junji Kobayashi, Department of General Medicine, Kanazawa Medical University, 1-1 Daigaku Uchinada, Kahoku, Ishikawa Prefecture 920-0293, Japan. Email: junjimaryland@gmail.com

doi: https://doi.org/10.14740/jocmr2806w

\section{Introduction}

It has been generally recognized that postprandial hyperglycemia and hyperlipidemia are highly related to the development of atherosclerosis [1-5]. Sodium/glucose cotransporter-2 (SGLT2) inhibitors have attracted attention as a new class of anti-diabetic agents for the treatment of type 2 diabetes [6-8]. The ultimate purpose of controlling blood glucose in diabetes is to prevent the development of its complication. Recently, it has been reported that empagliflozin, an SGLT2 inhibitor, significantly reduced rate of the primary composite cardiovascular outcome and of death in type 2 diabetes when added to standard care [9], suggesting this new class of anti-diabetes agents may prevent diabetes complication.

Tofogliflozin, another member of SGLT2 inhibitors, has been recently available in the market in Japan, with 2,900fold greater selectivity for SGLT2 than SGLT1, and has the highest selectivity of all clinically developed inhibitors [10]. Like other SGLT2 inhibitors [11], tofogliflozin treatment was associated with significant reductions in body weight (BW). These BW reductions might be associated with a compensatory reduction of visceral fat resulting from the loss of calories following increased urinary glucose excretion [12]. Indeed, a dapagliflozin study suggested that the main source of this BW reduction is visceral fat [13]. On the other hand, it is suggested that the accumulation of visceral fat contributes to the development of postprandial hyperlipidemia [14, 15], which is closely associated with cardiovascular disease [1-5].

In this background, we mainly focused on the effect of tofogliflozin treatment on lipid and lipoprotein metabolism in postprandial state using the cookie, an established meal [16] for the assessment of individuals' potential postprandial metabolic abnormalities in glucose and lipid.

\section{Materials and Methods}

\section{Study subjects}

Ten Japanese men with type 2 diabetes (average age 66.3 years) without any diabetes medication were orally administered tofogliflozin (20 mg per day) for 8 weeks followed by another 8 weeks of discontinuation (at 16 weeks). There were no sub- 


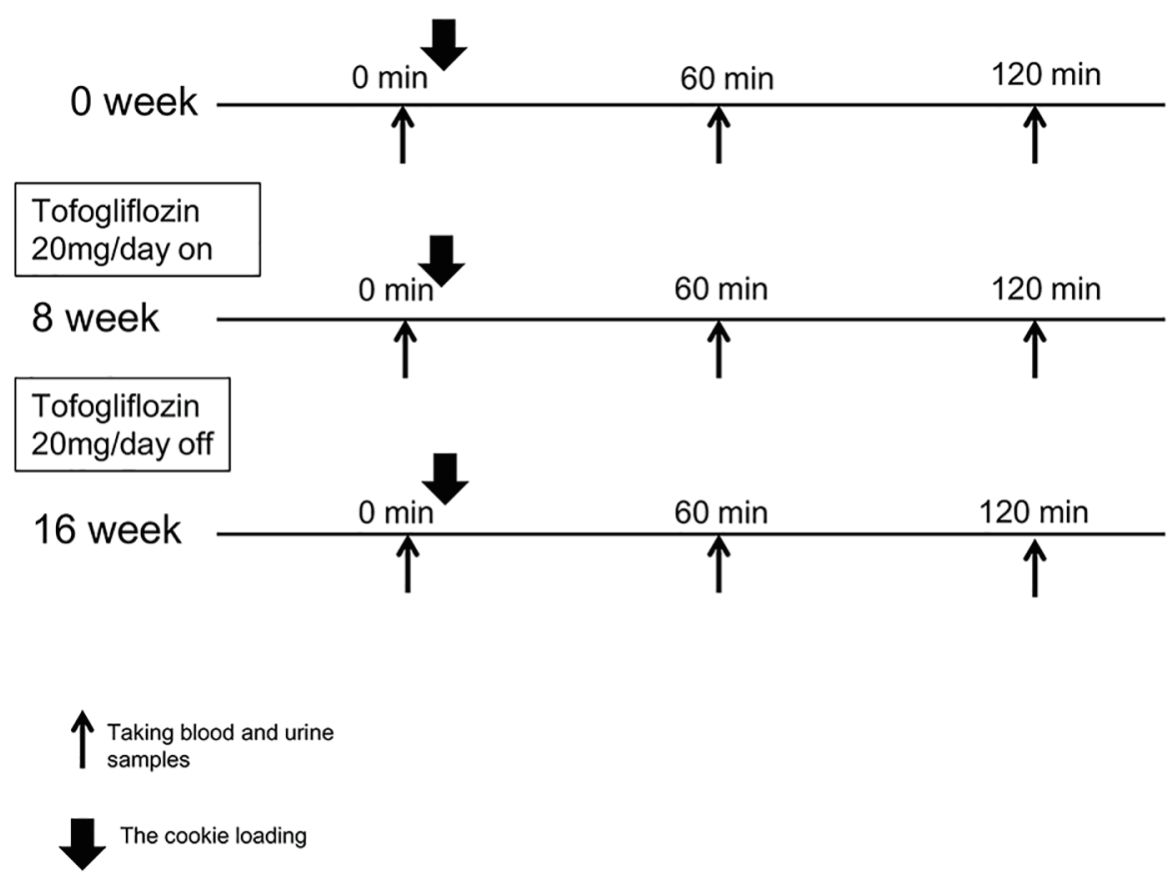

Figure 1. The study protocol is shown. We conducted the cookies tolerance test on three points: 0 week (baseline), 8 weeks (after treatment) and 16 weeks (after washout). Tofogliflozin (20 mg per day) administration started from the next day of the first cookies tolerance test and continued for 8 weeks. After 8 weeks of tofogliflozin administration and a subsequent 8-week washout of the agent, the cookies tolerance test was again conducted exactly the same way it was done before starting tofogliflozin.

jects with history of coronary artery disease or cerebrovascular disease. Five subjects were on antihypertensives medications and five subjects were on antihyperlipidemic agents. Doses of those drugs were not changed during this study period.

This work was conducted in accordance with Declaration of Helsinki. Informed consent was obtained from all of the participants. The institutional review board in Kanazawa Medical University Hospital approved the experimental protocol. This clinical trial was registered to UMIN and the registry number is UMIN000015778.

\section{Sample collection}

Study protocol is shown in Figure 1. This 16-week, singlearm, pilot clinical study investigated whether postprandial lipid metabolism would be improved after an 8-week oral administration of tofogliflozin ( $20 \mathrm{mg}$ per day) and a subsequent 8-week washout of the agent in Japanese men with type 2 diabetic patients. For the assessment of postprandial metabolism, we used the cookie (Saraya Corp., Osaka, Japan) [16], an established meal for the assessment of individuals' potential postprandial metabolic abnormalities in glucose and lipid. We conducted the cookies tolerance test on three points: 0 week (baseline), 8 weeks (after treatment) and 16 weeks (after washout).

On the morning of the day of examination, study subjects did urination completely and took $100 \mathrm{~mL}$ of water at $8 \mathrm{a} . \mathrm{m}$. and then did second urination right before taking blood sampling at 0 min followed by the oral ingestion of one pack of the cookie consisting of $75 \mathrm{~g}$ carbohydrate, $28.5 \mathrm{~g}$ fat, and $8 \mathrm{~g}$ protein for a total of $592 \mathrm{kcal}$. Subjects spent $10 \mathrm{~min}$ for taking the cookie with $120 \mathrm{~mL}$ of water and took blood and urine sampling at 60 and $120 \mathrm{~min}$. Tofogliflozin administration started from the next day for 8 weeks and then discontinued followed by another 8 weeks of observation without tofogliflozin.

\section{Measurement of metabolic parameters}

All laboratory tests were conducted by SRL Corporation, Japan. Cholesterol was measured by ultraviolet absorption spectrophotometry method and triglycerides (TG) were measured by GK-GPO free glycerol trap method.

HDL-C and LDL-C were measured by direct homogeneous assay methods using detergents. Quantification of remnant-like particles-cholesterol (RLP-C) and serum apoB-48 were conducted by the method using an immune-separation technique (Otsuka Pharmaceutical Co., Ltd) and a chemiluminescent enzyme assay (Fujirebio Co., Ltd), respectively. Immunoreactive insulin was measured by immunochemical methods. Measurement of the high-molecular weight (HMW) adiponectin was conducted by chemiluminescent enzyme assay (Fujirebio Co., Ltd). Serum lipoprotein lipase (LPL) mass was measured by ELISA (Sekisui Co., Ltd). Plasma glucagon levels were measured using a radioimmunoassay kit (Glucagon RIA (SML), Japan). Homeostasis model assessment-insulin resistance (HOMA-IR) was calculated as fasting insulin $\times$ fasting plasma glucose/405 and C-peptide index was calculated as C-peptide $\times 100 /$ fasting plasma glucose. 


\section{Analysis of postprandial lipid metabolism}

Metabolic parameters were measured at 0,60 and $120 \mathrm{~min}$ after the cookie loading. Results were presented as mean \pm SEM or medians (first and third quartiles). The area under the curve (AUC) of metabolic parameters was calculated by square measures of trapezoid on the basis of values obtained from 0 , 60 and 120 min after cookie ingestion.

The paired $t$ test was performed to assess the change from baseline. The Wilcoxon's signed rank test was performed when data were not distributed normally. A P value of less than 0.05 was considered to be significant.

\section{Results}

\section{Changes in glucose and related markers}

Changes in metabolic parameters related to glucose metabolism are shown in Table 1.

There were significant reductions in BW, body mass index (BMI) and waist circumference of the study subjects at 8 weeks vs. 0 week. At 8 weeks, there were significant reductions in plasma glucose (PG) at $60 \mathrm{~min}$ and $\mathrm{HbAlc}$, which returned to pretreatment level at 16 weeks. Serum insulin did not change during study period in either fasting or postprandial states. There were reductions in the AUC of PG and increases in that of plasma glucagon.

\section{Changes in lipids and related markers}

Changes in metabolic parameters related to lipid metabolism are shown in Table 2.

There were no significant changes in serum lipid and lipoprotein levels at 8 and 16 weeks vs. 0 week in either fasting or postprandial states except for tendency toward reduction in postprandial TG at 8 weeks and increase in HDL-C at 16 weeks.

There were no changes in apolipoprotein B48 during the study period either fasting or after test meal loading. There were no significant changes in the AUC of plasma TG throughout the study period.

\section{Changes in other parameters}

Changes in other metabolic parameters are shown in Table 3.

There were increases in red blood cells, hemoglobin and hematocrit at 8 weeks.

There were significant increases in serum HMW adiponectin levels. There were significant reductions in uric acid (UA) at 8 weeks, which returned to pretreatment levels at 16 weeks. There were tendency towards reductions in $\mathrm{uAlb} / \mathrm{Cr}$ after treatment.

There were no significant changes throughout study period in urinary $8-\mathrm{OHdG}$.

\section{Discussion}

The main findings of the current study are summarized as follows. 1) There were significant reductions in BW, BMI and waist circumference after tofogliflozin treatment. 2) There was a significant reduction in $\mathrm{HbA} 1 \mathrm{c}$ at 8 weeks, which returned to pretreatment levels at 16 weeks. 3) AUC of plasma glucagon significantly increased at 8 weeks. 4) There were no significant changes in lipid and lipoprotein levels in either fasting or postprandial state except for tendency toward reduction in postprandial TG at 8 weeks and increase in HDL-C at 16 weeks. 5) At 8 weeks, there was a significant reduction in UA, which returned to pretreatment levels at 16 weeks.

This is the first study on the effect of SGLT2 inhibitors treatment on lipid metabolism in postprandial states. In the past decade, increasing attention has been paid to the importance of postprandial TG as an important predictor to the development of atherosclerotic disease $[17,18]$. The current study, however, did not show significant changes in postprandial lipid metabolism during tofogliflozin treatment.

The findings that there were significant reductions in BW after tofogliflozin treatment are consistent with the previous studies $[19,20]$. Despite considerable reductions in BW during tofogliflozin treatment, there were no significant reductions in postprandial lipid and lipoproteins, which is in contrast to our findings [21] in anagliptin, a dipeptidyl peptidase-4 (DPP-4) inhibitor. Anagliptin treatment was associated with considerable reductions in postprandial dyslipidemia but not with significant reductions in $\mathrm{BW}$.

We assume that the potential contributing factor to offsetting BW reduction effect on postprandial lipidemia by tofogliflozin treatment could be the increases in glucagon. We found that in the current study tofogliflozin treatment was associated with increases in AUC of plasma glucagon, which is in line with recent reports on other SGLT2 inhibitors [22-24]. Glucagon, on the other hand, is known to increase hormone sensitive lipase activity in adipose tissue [25-27], leading to the increase in the influx of free fatty acid release into blood, which in turn may promote TG production from the liver. In contrast to SGLT2 inhibitors, studies suggest that plasma glucagon levels decreased during treatment with DPP-4 inhibitors [28-30].

Recent two studies have shown the clinical efficacy of tofogliflozin treatment in Japanese patients with type 2 diabetes mellitus $[19,20]$. One of the studies showed that there were increases in HDL-C but no significant changes in TC or LDL-C at 24 weeks by $20 \mathrm{mg}$ of tofogliflozin [19]. Another study showed that at 52 weeks, $20 \mathrm{mg}$ of tofogliflozin treatment caused increases in HDL-C and reductions in LDL-C but did not change TG [20]. These results suggest that the effects of tofogliflozin treatment on plasma lipid may not be straightforward. The recent study by Takahara et al [31] have shown a 4-week treatment with ipragliflozin, another SGLT2 inhibitor, in 10 Japanese type 2 diabetic adults produced no significant changes in plasma TG, total cholesterol, or HDL-C.

The limitations of this study are that the sample size is small and the study was done in a single arm. On the other hand, we conducted measurements of wide range of meta- 


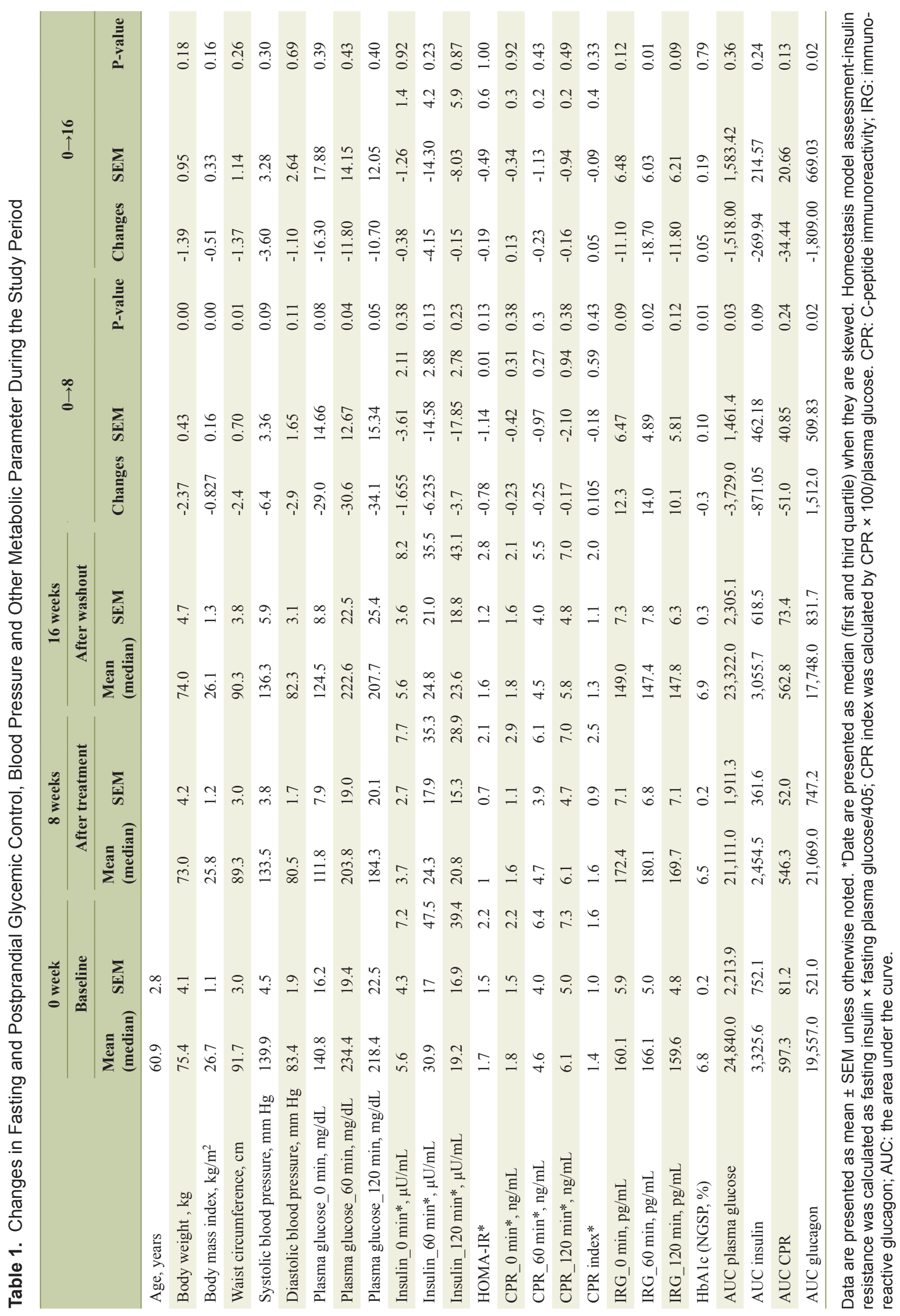



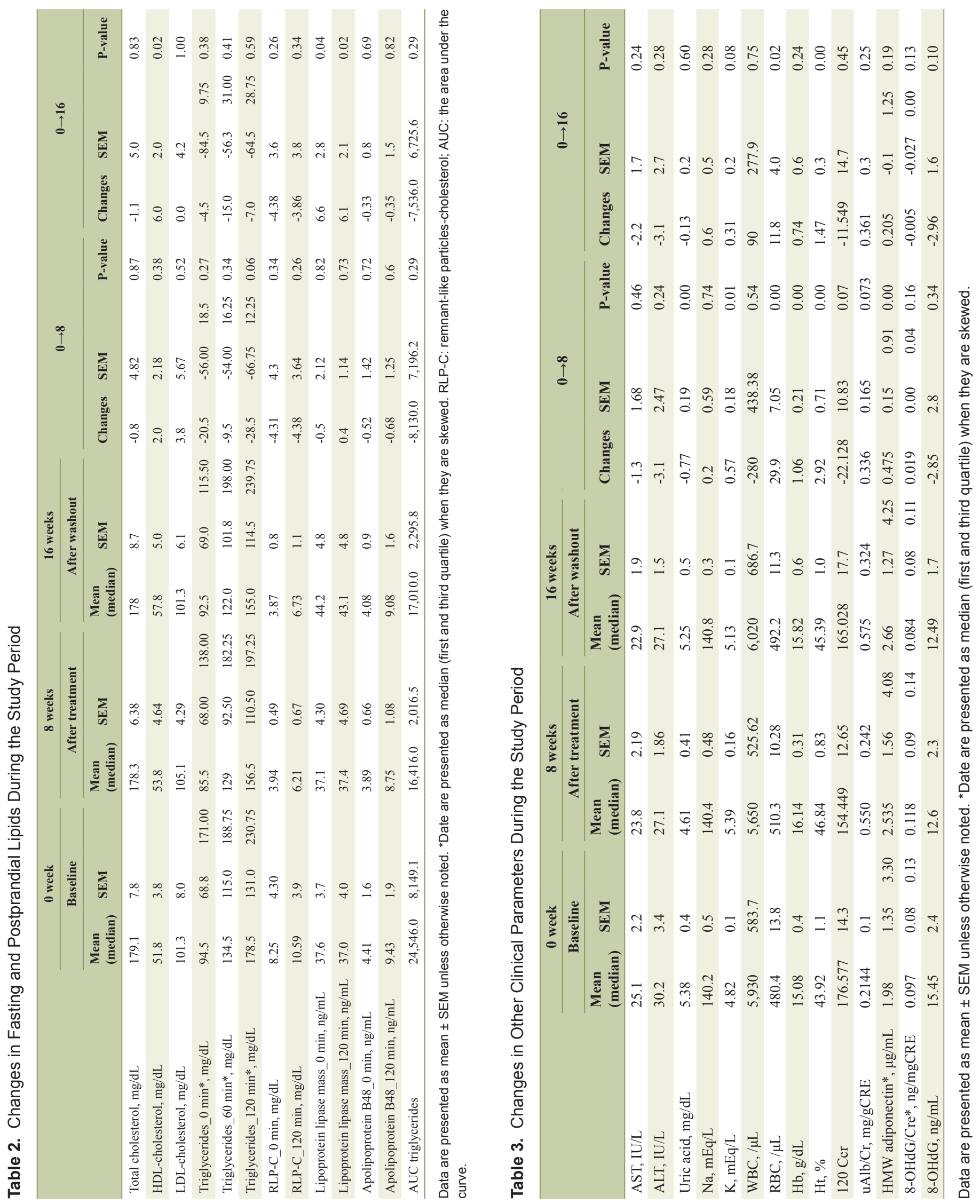
bolic markers in postprandial states as well as fasting during tofogliflozin treatment and after discontinuation of this treatment.

In conclusion, the present findings suggest that in men with type 2 diabetes, tofogliflozin treatment causes an improvement of postprandial glucose metabolisms but may not cause considerable changes in postprandial lipid and lipoprotein metabolism, which is in contrast to our previous findings in anagliptin, a DPP-4 inhibitor [21].

\section{Disclosure}

There is a conflict of interest regarding this study and this study was funded by Kowa Co., Ltd, Japan.

\section{References}

1. Glucose tolerance and cardiovascular mortality: comparison of fasting and 2-hour diagnostic criteria. Arch Intern Med. 2001;161(3):397-405.

2. Tominaga M, Eguchi H, Manaka H, Igarashi K, Kato T, Sekikawa A. Impaired glucose tolerance is a risk factor for cardiovascular disease, but not impaired fasting glucose. The Funagata Diabetes Study. Diabetes Care. 1999;22(6):920-924.

3. Zilversmit DB. Atherogenesis: a postprandial phenomenon. Circulation. 1979;60(3):473-485.

4. Karpe F. Postprandial lipoprotein metabolism and atherosclerosis. J Intern Med. 1999;246(4):341-355.

5. Havel RJ. Remnant lipoproteins as therapeutic targets. Curr Opin Lipidol. 2000;11(6):615-620.

6. Nair S, Wilding JP. Sodium glucose cotransporter 2 inhibitors as a new treatment for diabetes mellitus. J Clin Endocrinol Metab. 2010;95(1):34-42.

7. Vasilakou D, Karagiannis T, Athanasiadou E, Mainou M, Liakos A, Bekiari E, Sarigianni M, et al. Sodiumglucose cotransporter 2 inhibitors for type 2 diabetes: a systematic review and meta-analysis. Ann Intern Med. 2013;159(4):262-274.

8. Misra M. SGLT2 inhibitors: a promising new therapeutic option for treatment of type 2 diabetes mellitus. J Pharm Pharmacol. 2013;65(3):317-327.

9. Zinman B, Wanner C, Lachin JM, Fitchett D, Bluhmki E, Hantel S, Mattheus M, et al. Empagliflozin, Cardiovascular Outcomes, and Mortality in Type 2 Diabetes. N Engl J Med. 2015;373(22):2117-2128.

10. Suzuki M, Honda K, Fukazawa M, Ozawa K, Hagita H, Kawai T, Takeda M, et al. Tofogliflozin, a potent and highly specific sodium/glucose cotransporter 2 inhibitor, improves glycemic control in diabetic rats and mice. J Pharmacol Exp Ther. 2012;341(3):692-701.

11. Barnett $\mathbf{A H}$. Impact of sodium glucose cotransporter 2 inhibitors on weight in patients with type 2 diabetes mellitus. Postgrad Med. 2013;125(5):92-100.

12. Kleta R, Stuart C, Gill FA, Gahl WA. Renal glucosuria due to SGLT2 mutations. Mol Genet Metab. 2004;82(1):5658.
13. Bolinder J, Ljunggren O, Kullberg J, Johansson L, Wilding J, Langkilde AM, Sugg J, et al. Effects of dapagliflozin on body weight, total fat mass, and regional adipose tissue distribution in patients with type 2 diabetes mellitus with inadequate glycemic control on metformin. J Clin Endocrinol Metab. 2012;97(3):1020-1031.

14. Taira K, Hikita M, Kobayashi J, Bujo H, Takahashi K, Murano S, Morisaki N, et al. Delayed post-prandial lipid metabolism in subjects with intra-abdominal visceral fat accumulation. Eur J Clin Invest. 1999;29(4):301-308.

15. Couillard C, Bergeron N, Prud'homme D, Bergeron J, Tremblay A, Bouchard C, Mauriege P, et al. Postprandial triglyceride response in visceral obesity in men. Diabetes. 1998;47(6):953-960.

16. Harano Y, Miyawaki T, Nabiki J, Shibachi M, Adachi T, Ikeda M, Ueda F, et al. Development of cookie test for the simultaneous determination of glucose intolerance, hyperinsulinemia, insulin resistance and postprandial dyslipidemia. Endocr J. 2006;53(2):173-180.

17. Bansal S, Buring JE, Rifai N, Mora S, Sacks FM, Ridker PM. Fasting compared with nonfasting triglycerides and risk of cardiovascular events in women. JAMA. 2007;298(3):309-316.

18. Nordestgaard BG, Benn M, Schnohr P, Tybjaerg-Hansen A. Nonfasting triglycerides and risk of myocardial infarction, ischemic heart disease, and death in men and women. JAMA. 2007;298(3):299-308.

19. Kaku K, Watada H, Iwamoto Y, Utsunomiya K, Terauchi Y, Tobe K, Tanizawa Y, et al. Efficacy and safety of monotherapy with the novel sodium/glucose cotransporter-2 inhibitor tofogliflozin in Japanese patients with type 2 diabetes mellitus: a combined Phase 2 and 3 randomized, placebo-controlled, double-blind, parallel-group comparative study. Cardiovasc Diabetol. 2014;13:65.

20. Tanizawa Y, Kaku K, Araki E, Tobe K, Terauchi Y, Utsunomiya K, Iwamoto Y, et al. Long-term safety and efficacy of tofogliflozin, a selective inhibitor of sodium-glucose cotransporter 2, as monotherapy or in combination with other oral antidiabetic agents in Japanese patients with type 2 diabetes mellitus: multicenter, open-label, randomized controlled trials. Expert Opin Pharmacother. 2014;15(6):749-766.

21. Kakuda H, Kobayashi J, Kakuda M, Yamakawa J, Takekoshi N. The effect of anagliptin treatment on glucose metabolism and lipid metabolism, and oxidative stress in fasting and postprandial states using a test meal in Japanese men with type 2 diabetes. Endocrine. 2015;48(3):1005-1009.

22. Ferrannini E, Muscelli E, Frascerra S, Baldi S, Mari A, Heise T, Broedl UC, et al. Metabolic response to sodiumglucose cotransporter 2 inhibition in type 2 diabetic patients. J Clin Invest. 2014;124(2):499-508.

23. Merovci A, Solis-Herrera C, Daniele G, Eldor R, Fiorentino TV, Tripathy D, Xiong J, et al. Dapagliflozin improves muscle insulin sensitivity but enhances endogenous glucose production. J Clin Invest. 2014;124(2):509-514.

24. Bonner C, Kerr-Conte J, Gmyr V, Queniat G, Moerman $\mathrm{E}$, Thevenet J, Beaucamps $\mathrm{C}$, et al. Inhibition of the glucose transporter SGLT2 with dapagliflozin in pancre- 
atic alpha cells triggers glucagon secretion. Nat Med. 2015;21(5):512-517.

25. Slavin BG, Ong JM, Kern PA. Hormonal regulation of hormone-sensitive lipase activity and mRNA levels in isolated rat adipocytes. J Lipid Res. 1994;35(9):15351541.

26. Holm C, Kirchgessner TG, Svenson KL, Fredrikson G, Nilsson S, Miller CG, Shively JE, et al. Hormone-sensitive lipase: sequence, expression, and chromosomal localization to 19 cent-q13.3. Science. 1988;241(4872):15031506.

27. Stralfors P, Bjorgell P, Belfrage P. Hormonal regulation of hormone-sensitive lipase in intact adipocytes: identification of phosphorylated sites and effects on the phosphorylation by lipolytic hormones and insulin. Proc Natl Acad Sci U S A. 1984;81(11):3317-3321.

28. Solis-Herrera C, Triplitt C, Garduno-Garcia Jde J, Adams J, DeFronzo RA, Cersosimo E. Mechanisms of glucose lowering of dipeptidyl peptidase-4 inhibitor sitagliptin when used alone or with metformin in type 2 diabetes: a double-tracer study. Diabetes Care. 2013;36(9):27562762.

29. Murai K, Katsuno T, Miyagawa J, Matsuo T, Ochi F, Tokuda M, Kusunoki Y, et al. Very short-term effects of the dipeptidyl peptidase-4 inhibitor sitagliptin on the secretion of insulin, glucagon, and incretin hormones in Japanese patients with type 2 diabetes mellitus: analysis of meal tolerance test data. Drugs R D. 2014;14(4):301308.

30. Moritoh Y, Takeuchi K, Asakawa T, Kataoka O, Odaka H. Chronic administration of alogliptin, a novel, potent, and highly selective dipeptidyl peptidase-4 inhibitor, improves glycemic control and beta-cell function in obese diabetic ob/ob mice. Eur J Pharmacol. 2008;588(23):325-332.

31. Takahara M, Shiraiwa T, Matsuoka TA, Katakami N, Shimomura I. Ameliorated pancreatic beta cell dysfunction in type 2 diabetic patients treated with a sodiumglucose cotransporter 2 inhibitor ipragliflozin. Endocr J. 2015;62(1):77-86. 